\title{
PERTURBATION METHOD FOR LINEAR DIFFERENCE EQUATIONS WITH SMALL PARAMETERS
}

\author{
TAHIA ZERIZER
}

Received 14 December 2005; Revised 5 April 2006; Accepted 26 April 2006

We consider a boundary value problem for a linear difference equation with several widely different coefficients. We study the existence and uniqueness of its solution and we give successive asymptotic approximations for this solution, obtained by a simple iterative method. This method improves the singular perturbation method, it offers considerable reduction and simplicity in computation since it does not require to compute boundary layer correction solutions.

Copyright (C 2006 Tahia Zerizer. This is an open access article distributed under the Creative Commons Attribution License, which permits unrestricted use, distribution, and reproduction in any medium, provided the original work is properly cited.

\section{Introduction}

In recent years, several methods have been developed for the study of boundary value problems for difference equations, see, for example, $[2,3,8]$. In this paper, we consider the $(m+n)$ th-order difference equation:

$$
\begin{array}{r}
\varepsilon^{m} a_{m+n, k} y_{k+n+m}+\cdots+\varepsilon^{2} a_{n+2, k} y_{k+n+2}+\varepsilon a_{n+1, k} y_{k+n+1} \\
+a_{n, k} y_{k+n}+a_{n-1, k} y_{k+n-1}+\cdots+a_{1, k} y_{k+1}+a_{0, k} y_{k}=f_{k}, \\
k=0,1, \ldots, N-n-m,
\end{array}
$$

where $\varepsilon$ is a small parameter, $\left(a_{i, k}\right), 0 \leq i \leq m+n,\left(f_{k}\right), 0 \leq k \leq N-n-m$, are given discrete real functions, and $N$ is a fixed integer. We associate to (1.1) the boundary conditions

$$
y_{k}=\alpha_{k}, \quad k=0, \ldots, n-1, \quad y_{N-k}=\beta_{k}, \quad k=0, \ldots, m-1,
$$

where $\alpha_{k}, 0 \leq k \leq n-1$, and $\beta_{k}, 0 \leq k \leq m-1$, are given constants.

We are concerned with the boundary value problem $\left(P_{\varepsilon}\right)$ described by $(1.1)$ and (1.2), it is a classical representation of multi-time-scale digital systems. Such systems are 
prevalent in engineering and other great applications especially digital control theory [9$11,14]$ and their perturbation analysis is gaining momentum $[4-7,13]$. The presence of small parameters increases the order of the system and exhibits time-scale phenomena. The high dimensionality coupled with the time-scale behavior makes the system computationally stiff resulting in the use of extensive numerical routines.

Recently, the particular case $m=1, n=1$ of problem $\left(P_{\varepsilon}\right)$ was studied in [15]. Through this paper, we studied the existence and uniqueness of the solution and we developed an iterative convergent method to get successive asymptotic approximations for this solution.

The boundary problem $\left(P_{\varepsilon}\right)$ was also considered by Comstock and Hsiao [1] in the homogeneous case $f_{k}=0$ for $m=1, n=1$. The time-invariant case of problem $\left(P_{\varepsilon}\right)$ was considered by Naidu and Rao (see [12, Chapter 1]) and also by Krishnarayalu [7], where small parameters are multiple. These authors developed a singular perturbation method, a formal procedure, to give approximate solutions which consist of outer solutions and boundary layer correction solutions. Notice that in general this method cannot be extended for the general case of time-variant problems (see Section 3.2, Remark 3.3, for the arguments).

The aim of this paper is to extend for problem $\left(P_{\varepsilon}\right)$ the perturbation method developed in [15]. We give sufficient conditions on the coefficients of (1.1), to ensure existence and uniqueness of the solution of problem $\left(P_{\varepsilon}\right)$, and successive approximations of this solution, obtained by a simple procedure. A proof is given of uniform convergence of the iterative method. The most distinguished feature of this method, besides order reduction, is the decoupling of the original boundary value problem into initial value problems, which facilitates considerable treatment of the boundary value problem. The proposed method consists simply of writing the problem $\left(P_{\varepsilon}\right)$ in a matrix form (see the proof of Theorem 2.1 for the details), and can be easily applied to initial value problems.

Our method is proposed to improve the singular perturbation method, it offers considerable reduction and simplicity in computation because it does not require to compute boundary layer correction solutions. The difference between both methods lies in the definition of boundary conditions of the degenerate system, obtained by suppressing the perturbation parameter in the initial system (1.1)-(1.2).

The remainder of the paper is organized as follows. In Section 2, we give the main results. We study the existence and uniqueness of the problem $\left(P_{\varepsilon}\right)$, we present our procedure to get approximate solutions, and we give proof of uniform convergence of the proposed iterative method. Section 3 is mainly devoted to validate the effectiveness of our method, compared with the singular perturbation method. We consider a right end perturbation (small parameters are situated on the right), and we deduce the results from Section 2. The comparison with the other formal method requires the analysis of its results. We conclude with Section 4.

\section{Main result}

2.1. Formal asymptotic solution. In this section, we develop a perturbation method to obtain asymptotic approximate solutions for the whole order. This iterative method facilitates a considerable reduction and simplicity in computation. Like in any perturbation 
method, the solution $y_{k}(\varepsilon), 0 \leq k \leq N$, of problem $\left(P_{\varepsilon}\right)$ is assumed as a power series in $\varepsilon$, we seek for a solution of the natural form

$$
y_{k}(\varepsilon)=\sum_{j=0}^{\infty} \varepsilon^{j} y_{k}^{(j)}, \quad 0 \leq k \leq N .
$$

Substituting the formal expansion (2.1) into (1.1)-(1.2), and equating the coefficients at the same powers of $\varepsilon$, a set of equations is obtained. For the zeroth-order asymptotic approximation, the resulting equations are given by

$$
\begin{gathered}
y_{k}^{(0)}=\alpha_{k}, \quad k=0,1, \ldots, n-1, \\
a_{n, k} y_{k+n}^{(0)}+a_{n-1, k} y_{k+n-1}^{(0)}+\cdots+a_{0, k} y_{k}^{(0)}=f_{k}, \quad k=0,1, \ldots, N-m-n, \\
y_{N-k}^{(0)}=\beta_{k}, \quad k=0,1, \ldots, m-1 .
\end{gathered}
$$

The system described by (2.2) corresponds to the degenerate problem of $\left(P_{\varepsilon}\right)$, it is obtained by suppressing the perturbation parameter in (1.1)-(2.1). Notice that (2.2) is an initial value problem. It defines the sequence $\left(y_{0}^{(0)}, \ldots, y_{N-m}^{(0)}\right)$ if and only if $a_{n, k} \neq 0$ for $0 \leq k \leq$ $N-n-m$. The final conditions in (2.2) define the sequence $\left(y_{N-m+1}^{(0)}, \ldots, y_{N}^{(0)}\right)$.

The terms $y_{k}^{(0)}, 0 \leq k \leq N-n-m$, can be computed without any knowledge of the boundary conditions $y_{N-k}=\beta_{k}, 0 \leq k \leq m-1$. By analogy with the case of differential equations, we can say that there are $m$ boundary layers located at the right, that is, at the final values.

For the $j$ th-order asymptotic approximation, $j \geq 1$, we agree that

$$
y_{k}^{(l)} \equiv 0, \quad l<0,0 \leq k \leq N
$$

to give a compact writing of the resulting equations which are given by

$$
\begin{gathered}
y_{k}^{(j)}=0, \quad k=0,1, \ldots, n-1, \\
a_{n, k} y_{k+n}^{(j)}+a_{n-1, k} y_{k+n-1}^{(j)}+\cdots+a_{0, k} y_{k}^{(j)} \\
=-a_{n+1, k} y_{k+n+1}^{(j-1)}-a_{n+2, k} y_{k+n+2}^{(j-2)} \\
-\cdots-a_{n+m, k} y_{k+n+m}^{(j-m)}, \quad k=0, \ldots, N-n-m, \\
y_{N-k}^{(j)}=0, \quad k=0,1, \ldots, m-1 .
\end{gathered}
$$

Notice that $(2.4)$ is an initial value problem. It defines the sequence $\left(y_{0}^{(j)}, \ldots, y_{N-m}^{(j)}\right)$ if and only if $a_{n, k} \neq 0$ for $0 \leq k \leq N-n-m$. The final conditions in (2.4) define the sequence $\left(y_{N-m+1}^{(j)}, \ldots, y_{N}^{(j)}\right)$.

2.2. Existence and convergence. In this section, we present the main results of this paper. We give sufficient conditions that guarantee, for problem $\left(P_{\varepsilon}\right)$, existence and uniqueness 
4 Perturbation method for difference equations

of the solution, and we prove the convergence of the series (2.1). The following theorem includes these results.

Theorem 2.1. Assume that $a_{n, k} \neq 0$ for $0 \leq k \leq N-n-m$. Then there exists positive real number $\varepsilon_{0}$, if $|\varepsilon|<\varepsilon_{0}$, the solution $\left(y_{k}(\varepsilon)\right)$ of $\left(P_{\varepsilon}\right)$ exists and is unique and satisfies (2.1) uniformly for $0 \leq k \leq N$, where $y_{k}^{(0)}$ and $y_{k}^{(j)}$ are the solutions of (2.2) and (2.4), respectively. More precisely, for all $n \geq 0$ and all $0 \leq k \leq N$,

$$
\left|y_{k}(\varepsilon)-\sum_{j=0}^{n} \varepsilon^{j} y_{k}^{(j)}\right| \leq C \frac{\left(|\varepsilon| / \varepsilon_{0}\right)^{n+1}}{1-|\varepsilon| / \varepsilon_{0}}
$$

where $C$ is a constant independent of $n$ and $\varepsilon$.

Proof. For all $k=0,1, \ldots, N-n-m$, we use the transformations

$$
\begin{gathered}
u_{1}(k+n+2)=\varepsilon y_{k+n+2}, \\
u_{2}(k+n+3)=\varepsilon u_{1}(k+n+3), \\
\cdots \\
u_{m-1}(k+n+m)=\varepsilon u_{m-2}(k+n+m),
\end{gathered}
$$

then problem $\left(P_{\varepsilon}\right)$ becomes

$$
\begin{gathered}
y_{k}=\alpha_{k}, \quad k=0,1, \ldots, n-1, \\
\varepsilon\left(a_{m+n, k} u_{m-1}(k+m+n)+\cdots+a_{n+2, k} u_{1}(k+n+2)+a_{n+1, k} y_{k+n+1}\right) \\
+a_{n, k} y_{k+n}+a_{n-1, k} y_{k+n-1}+\cdots+a_{0, k} y_{k}=f_{k}, \quad k=0,1, \ldots, N-n-m, \\
y_{N-k}=\beta_{k}, \quad k=0,1, \ldots, m-1 .
\end{gathered}
$$

We can now write the system (2.7) in the matrix form

$$
A_{\varepsilon} y=\left(A_{0}+\varepsilon U\right) y=f
$$

where

$$
\begin{gathered}
y=\left(y_{0}, y_{1}, \ldots, y_{N}, u_{1}, \ldots, u_{m-1}\right)^{t}, \\
u_{j}=\left(u_{j}(n+j+1), \ldots, u_{j}(N-m+j+1)\right)^{t}, \quad j=1, \ldots, m-1, \\
f=\left(\alpha_{0}, \alpha_{1}, \ldots, \alpha_{n-1}, f_{0}, f_{1}, \ldots, f_{N-n-m}, \beta_{m-1}, \beta_{m-2}, \ldots, \beta_{0}, 0, \ldots, 0\right),
\end{gathered}
$$


and the matrix $A_{0}$ is given by

$$
\left(\begin{array}{ccccccccc}
1 & \cdots & 0 & 0 & \cdots & 0 & 0 & \cdots & 0 \\
0 & \cdots & 0 & 0 & \cdots & 0 & 0 & \cdots & 0 \\
\vdots & \ddots & \vdots & \vdots & & \vdots & \vdots & & \vdots \\
0 & \cdots & 1 & 0 & \cdots & 0 & 0 & \cdots & 0 \\
a_{0,0} & \cdots & a_{n-1,0} & a_{n, 0} & \cdots & 0 & 0 & \cdots & 0 \\
\vdots & \ddots & \vdots & \vdots & \ddots & \vdots & \vdots & & \vdots \\
0 & \cdots & 0 & a_{0, N-n-m} & \cdots & a_{n, N-n-m} & 0 & \cdots & 0 \\
0 & \cdots & 0 & 0 & \cdots & 0 & 1 & \cdots & 0 \\
\vdots & & \vdots & \vdots & & \vdots & \vdots & \ddots & \vdots \\
0 & \cdots & 0 & 0 & \cdots & 0 & 0 & \cdots & 1
\end{array}\right)
$$

whereas matrixes $A_{\varepsilon}$ and $U$ can be deduced easily from (2.7), (2.8), (2.9), and (2.10). We indifferently denote by $\|\cdot\|$ the infinity norm in $\mathbb{R}^{N+1+(m-1) \times(N-n-m+1)}$ and the associated matrix norm. Since $a_{n, k} \neq 0$ for $0 \leq k \leq N-n-m$, matrix $A_{0}$ is nonsingular and we can define the positive number

$$
\varepsilon_{0}=\frac{1}{\left\|U A_{0}^{-1}\right\|}
$$

If $|\varepsilon|<\varepsilon_{0}$, we deduce from (2.8) that

$$
A_{0}^{-1} \sum_{l=0}^{+\infty}\left(-\varepsilon U A_{0}^{-1}\right)^{l}=A_{0}^{-1}\left(I+\varepsilon U A_{0}^{-1}\right)^{-1}=A_{\varepsilon}^{-1},
$$

that is, the inverse of matrix $A_{\varepsilon}$ is well defined for $|\varepsilon|<\varepsilon_{0}$, and (2.8) has a unique solution $y(\varepsilon)$ given by

$$
y(\varepsilon)=A_{\varepsilon}^{-1} f .
$$

We denote

$$
\begin{gathered}
y^{(l)}=A_{0}^{-1}\left(-U A_{0}^{-1}\right)^{l} f, \quad l \geq 0, \\
y^{(l)}=\left(y_{0}^{(l)}, y_{1}^{(l)}, \ldots, y_{N}^{(l)}, u_{1}^{(l)}, \ldots, u_{m-1}^{(l)}\right)^{t} \in \mathbb{R}^{N+1+(m-1) \times(N-n-m+1)}, \quad y_{k}^{(l)} \in \mathbb{R}, \\
u_{j}^{(l)}=\left(u_{j}^{(l)}(n+j+1), \ldots, u_{j}^{(l)}(N-m+j+1)\right)^{t}, \quad j=1, \ldots, m-1,
\end{gathered}
$$

from (2.12), (2.13), and (2.14) we deduce that, for $|\varepsilon|<\varepsilon_{0}$, the solution $y(\varepsilon)$ of (2.8) can be represented in the convergent series:

$$
y(\varepsilon)=\sum_{l=0}^{+\infty} \varepsilon^{l} y^{(l)}
$$




\section{Perturbation method for difference equations}

We can easily verify that the $N+1$ first components of $y^{(0)}$ (resp., $y^{(l)}, l \geq 1$ ), that is, $y_{k}^{(0)}$, $0 \leq k \leq N$, (resp., $y_{k}^{(l)}, 0 \leq k \leq N, l \geq 1$ ) satisfy problem (2.2) (resp., problem (2.4)).

To prove estimate (2.5), we compute the remainder of series (2.12),

$$
\begin{gathered}
\left\|A_{\varepsilon}^{-1}-A_{0}^{-1} \sum_{l=0}^{n}\left(-\varepsilon U A_{0}^{-1}\right)^{l}\right\| \leq\left\|A_{0}^{-1}\right\| \sum_{l=n+1}^{+\infty}\left\|\varepsilon U A_{0}^{-1}\right\|^{l} \\
=\frac{\left\|A_{0}^{-1}\right\|\left\|\varepsilon U A_{0}^{-1}\right\|^{n+1}}{1-\left\|\varepsilon U A_{0}^{-1}\right\|} \leq\left\|A_{0}^{-1}\right\| \frac{\left(|\varepsilon| / \varepsilon_{0}\right)^{n+1}}{1-|\varepsilon| / \varepsilon_{0}} .
\end{gathered}
$$

We denote by $C$ the real positive number

$$
C=\left\|A_{0}^{-1}\right\|\|f\|
$$

we deduce from (2.13), (2.17), and (2.18) that

$$
\left\|y(\varepsilon)-\sum_{j=0}^{n} \varepsilon^{j} y^{(j)}\right\| \leq C \frac{\left(|\varepsilon| / \varepsilon_{0}\right)^{n+1}}{1-|\varepsilon| / \varepsilon_{0}},
$$

with the chosen norm, we obtain (2.5).

\section{Comparison with singular perturbation method}

In this section, we consider a right end perturbation [12]. We are concerned with the boundary value problem

$$
\begin{gathered}
y_{k}=\alpha_{k}, \quad k=0, \ldots, m-1, \\
a_{m+n, k} y_{k+m+n}+\cdots+a_{m+1, k} y_{k+m+1}+a_{m, k} y_{k+m} \\
+\varepsilon a_{m-1, k} y_{k+m-1}+\cdots+\varepsilon^{m-1} a_{1, k} y_{k+1}+\varepsilon^{m} a_{0, k} y_{k} \\
=f_{k}, \quad k=0,1, \ldots, N-n-m, \\
y_{N-k}=\beta_{k}, \quad k=0, \ldots, n-1 .
\end{gathered}
$$

The stationary case of problem (3.1) was considered in [12]. The singular perturbation method was developed to get asymptotic expansions for the solution.

In order to validate the effectiveness of our method, we compare the results given by both methods. First, we give our results which are easy to deduce from Section 2, then we analyze the formal expansions obtained in [12].

3.1. Perturbation method. Using the transformation

$$
z_{k}=y_{N-k}, \quad 0 \leq k \leq N,
$$

problem (3.1) is brought in the form (1.1)-(1.2) and we can already state the results. 
Let $y_{k}^{(0)}, 0 \leq k \leq N$, be the solution of the problem

$$
\begin{gathered}
y_{k}^{(0)}=\alpha_{k}, \quad k=0,1, \ldots, m-1, \\
a_{m+n, k} y_{k+m+n}^{(0)}+\cdots+a_{m+1, k} y_{k+m+1}^{(0)}+a_{m, k} y_{k+m}^{(0)} \\
=f_{k}, \quad k=0,1, \ldots, N-m-n, \\
y_{N-k}^{(0)}=\beta_{k}, \quad k=0,1, \ldots, n-1 .
\end{gathered}
$$

The sequence $\left(y_{0}^{(0)}, \ldots, y_{N-m}^{(0)}\right)$ in $(3.3)$ can be computed using the final values $y_{m}^{(0)}, \ldots, y_{N}^{(0)}$ if and only if $a_{m, k} \neq 0$ for $0 \leq k \leq N-n-m$. The values $y_{0}^{(0)}, \ldots, y_{m-1}^{(0)}$ are fixed. The $m$ boundary layers are located at the left, at the initial values.

Once the coefficients $y_{k}^{(0)}, 0 \leq k \leq N$, are fixed from (3.3), we can define the following problems recursively for $j \geq 1$.

Let $y_{k}^{(j)}, 0 \leq k \leq N, j \geq 1$, be the solution of the problem

$$
\begin{gathered}
y_{k}^{(j)}=0, \quad k=0,1, \ldots, m-1, \\
a_{m+n, k} y_{k+m+n}^{(j)}+\cdots+a_{m+1, k} y_{k+m+1}^{(j)}+a_{m, k} y_{k}^{(j)} \\
=-a_{m-1, k} y_{k}^{(j-1)}-a_{m-2, k} y_{k}^{(j-2)}-\cdots \\
-a_{0, k} y_{k}^{(j-m)}, \quad k=0,1, \ldots, N-m-n, \\
y_{N-k}^{(j)}=0, \quad k=0,1, \ldots, n-1 .
\end{gathered}
$$

The sequence $\left(y_{0}^{(j)}, \ldots, y_{N-m}^{(j)}\right)$ can be computed using the final values $y_{N-n+1}^{(j)}, \ldots, y_{N}^{(j)}$ in (3.4) if and only if $a_{m, k} \neq 0$ for $0 \leq k \leq N-n-m$. The values $y_{0}^{(j)}, \ldots, y_{m-1}^{(j)}$ are fixed.

Corollary 3.1. Assume that $a_{m, k} \neq 0$ for $0 \leq k \leq N-n-m$. Then there exists positive real number $\varepsilon_{0}$, if $|\varepsilon|<\varepsilon_{0}$, the solution $\left(y_{k}(\varepsilon)\right)$ of (3.1) exists and is unique, and satisfies

$$
y_{k}(\varepsilon)=\sum_{j=0}^{\infty} \varepsilon^{j} y_{k}^{(j)}, \quad 0 \leq k \leq N,
$$

uniformly for $0 \leq k \leq N$, where $y_{k}^{(0)}$ and $y_{k}^{(j)}$ are the solutions of (2.2) and (2.4), respectively. More precisely, for all $n \geq 0$ and all $0 \leq k \leq N$,

$$
\left|y_{k}(\varepsilon)-\sum_{j=0}^{n} \varepsilon^{j} y_{k}^{(j)}\right| \leq C \frac{\left(|\varepsilon| / \varepsilon_{0}\right)^{n+1}}{1-|\varepsilon| / \varepsilon_{0}}
$$

where $C$ is a constant independent of $n$ and $\varepsilon$.

Proof. A direct consequence of (3.2) and Theorem 2.1. 
8 Perturbation method for difference equations

3.2. Singular perturbation method. The following problem:

$$
\begin{gathered}
y_{k}=\alpha_{k}, \quad k=0, \ldots, m-1, \\
y_{k+m+n}+\cdots+a_{m} y_{k+m}+\varepsilon a_{m-1} y_{k+m-1}+\cdots+\varepsilon^{m} a_{0} y_{k} \\
=f_{k}, \quad k=0,1, \ldots, N-n-m, \\
y_{N-k}=\beta_{k}, \quad k=0, \ldots, n-1,
\end{gathered}
$$

was considered in [12], it corresponds to the stationary case of problem (3.1). By analogy with the case of ordinary differential equations, the authors developed a singular perturbation method. They wrote the solution $y_{k}(\varepsilon), 0 \leq k \leq N$, of (3.7) in the form

$$
y_{k}(\varepsilon)=\sum_{j=0}^{\infty} \varepsilon^{j} y_{t, k}^{(j)}+\varepsilon^{k} \sum_{j=0}^{\infty} \varepsilon^{j} w_{0, k}^{(j)}+\cdots+\varepsilon^{k-m+1} \sum_{j=0}^{\infty} \varepsilon^{j} w_{m-1, k}^{(j)}, \quad 0 \leq k \leq N,
$$

where $\sum_{j=0}^{\infty} \varepsilon^{j} y_{t, k}^{(j)}$ is the outer series and $\sum_{j=0}^{\infty} \varepsilon^{j} w_{s, k}^{(j)}, s=0, \ldots, m-1$, is the correction series introduced to recover the $m$ boundary layers located in the initial conditions.

The coefficients $y_{t, k}^{(0)}$ are the solutions of the final value problem (3.9)-(3.10),

$$
\begin{gathered}
a_{m+n} y_{t, k+m+n}^{(0)}+a_{m+n-1} y_{t, k+m+n-1}^{(0)}+\cdots+a_{m} y_{t, k+m}^{(0)} \\
=f_{k}, \quad k=N-n-m, N-n-m-1, \ldots, \\
y_{t, N}^{(0)}=\beta_{0}, y_{t, N-1}^{(0)}=\beta_{1}, \ldots, y_{t, N-n+1}^{(0)}=\beta_{n-1} .
\end{gathered}
$$

The coefficients $w_{s, k}^{(0)}, s=0,1, \ldots, m-1$, are the solutions of the initial value problem (3.11)-(3.12),

$$
\begin{gathered}
a_{m} w_{s, k+m}^{(0)}+a_{m-1} w_{s, k+m-1}^{(0)}+\cdots+a_{0} w_{s, k}^{(0)}=0, \quad k=0,1, \ldots, \\
w_{s, s}^{(0)}=\alpha_{s}-y_{t, s}^{(0)}, \quad w_{s, k}^{(0)}=0 \quad \text { if } k \neq s, s=0,1, \ldots, m-1 .
\end{gathered}
$$

To give a single writing for the problems which define the coefficients $y_{t, k}^{(j)}, w_{s, k}^{(j)}, j \geq 1$, $0 \leq s \leq m-1$, we agree that for all $k, 0 \leq s \leq m-1, j<0$,

$$
y_{t, k}^{(j)} \equiv 0, \quad w_{s, k}^{(j)} \equiv 0 .
$$

The coefficients $y_{t, k}^{(j)}$ are the solutions of the final value problem (3.14)-(3.15),

$$
\begin{gathered}
a_{m+n} y_{t, k+m+n}^{(j)}+a_{m+n-1} y_{t, k+m+n-1}^{(j)}+\cdots+a_{m} y_{t, k+m}^{(j)} \\
=-a_{m-1} y_{t, k+m-1}^{(j-1)}-a_{m-2} y_{t, k+m-2}^{(j-2)}-\cdots \\
-a_{0} y_{t, k}^{(j-m)}, \quad k=N-n-m, N-n-m-1, \ldots, \\
y_{t, N}^{(j)}=y_{t, N-1}^{(j)}=\cdots=y_{t, N-n+1}^{(j)}=0 .
\end{gathered}
$$


The coefficients $w_{s, k}^{(j)}, 0 \leq s \leq m-1, j \geq 1$, are the solutions of the initial value problem (3.16)-(3.17),

$$
\begin{gathered}
a_{m} w_{s, k+m}^{(j)}+a_{m-1} w_{s, k+m-1}^{(j)}+\cdots+a_{0} w_{s, k}^{(j)} \\
=-a_{m+1} w_{s, k+m+1}^{(j-1)}-a_{m+2} w_{s, k+m+2}^{(j-2)}-\cdots \\
\quad-a_{m+n} w_{s, k+m+n}^{(j-n)}, \quad k=0,1, \ldots, \\
w_{s, s}^{(j)}=-y_{t, s}^{(j)}, \quad w_{s, k}^{(j)}=0 \quad \text { if } k \neq s, 0 \leq s \leq m-1 .
\end{gathered}
$$

This formal procedure was not justified. The expansion (3.8) is not asymptotic when the order is equal to $N-n-m+2$, see the following proposition.

Proposition 3.2. The series (3.19) is not an asymptotic expansion of the solution $y_{k}(\varepsilon)$ of $\operatorname{order} N-n-m+2$.

Proof. Since $\tilde{y}_{N-n+1}^{(0)}=\beta_{n-1}$ and $y_{t, N-n+1}^{(j)}=0$ for $j \geq 1$, from (3.8) we get

$$
y_{N-n+1}(\varepsilon)=\beta_{n-1}+\varepsilon^{N-n-m+2} w_{m-1, N-n+1}^{(0)}+\cdots .
$$

Since in general $w_{m-1, N-n+1}^{(0)} \neq 0,(3.8)$ is not an asymptotic expansion of $y_{N-n+1}(\varepsilon)=\beta_{n-1}$ of order $N-n-m+2$.

Remark 3.3. To get the correction terms $w_{s, k}^{(j)}, 0 \leq s \leq m-1, j \geq 0$, we must compute the values $y_{t, 0}^{(0)}, y_{t, 1}^{(0)}, \ldots, y_{t, m-1}^{(0)}$ and $y_{t, 0}^{(j)}, y_{t, 1}^{(j)}, \ldots, y_{t, m-1}^{(j)}$. This computation requires to solve the difference equations (3.9) and (3.14) for $k=-1,-2, \ldots,-m$. In general, for the timevariant case, we do not have at our disposal the values $a_{m,-l}, a_{m+1,-l}, \ldots, a_{m+n-1,-l}, f_{-l}, l=$ $1,2, \ldots, m$ which allow us to compute the coefficients $y_{t, s}^{(0)}$ and $y_{t, s}^{(j)}, j \geq 1$. Consequently, we cannot define the problems (3.11)-(3.12) and (3.16)-(3.17).

3.2.1. There is no need of correction series. In this section, we compare the expansion (3.8) and our expansion given in Section 3.1, for the time-invariant case. From (3.8) we see that

$$
y_{k}(\varepsilon)=\sum_{j=0}^{+\infty} \varepsilon^{j} \tilde{y}_{k}^{(j)}, \quad 0 \leq k \leq N,
$$

where

$$
\tilde{y}_{k}^{(j)}=y_{t, k}^{(j)}+w_{0, k}^{(j-k)}+w_{1, k}^{(j-k+1)}+\cdots+w_{m-1, k}^{(j-k+m-1)}, \quad 0 \leq k \leq N, j \geq 0 .
$$

Proposition 3.4. The coefficients of series (3.19) satisfy

$$
\begin{gathered}
\tilde{y}_{s}^{(0)}=\alpha_{s}, \quad 0 \leq s \leq m-1, \quad \tilde{y}_{k}^{(0)}=\beta_{N-k}, \quad N-n+1 \leq k \leq N, \\
\tilde{y}_{s}^{(j)}=0, \quad 0 \leq s \leq m-1, \quad \tilde{y}_{k}^{(j)}=0, \quad N-n+1 \leq k \leq N, 1 \leq j \leq N-n-m+1 .
\end{gathered}
$$


Proof. From (3.12) and (3.20), we deduce

$$
\tilde{y}_{s}^{(0)}=y_{t, s}^{(0)}+w_{0, s}^{(-s)}+w_{1, s}^{(-s+1)}+\cdots+w_{m-1, s}^{(-s+m-1)}=y_{t, s}^{(0)}+w_{s, s}^{(0)}=\alpha_{s}, \quad s=0,1, \ldots, m-1 .
$$

Equations (3.10) and (3.20) give

$$
\tilde{y}_{k}^{(0)}=y_{t, k}^{(0)} \equiv \beta_{N-k}, \quad k=N-n+1, N-n+2, \ldots, N .
$$

In addition, (3.17) and (3.20) give

$$
\tilde{y}_{s}^{(j)}=y_{t, s}^{(j)}+w_{0, s}^{(j-s)}+w_{1, s}^{(j-s+1)}+\cdots+w_{m-1, s}^{(j-s+m-1)}=y_{t, s}^{(j)}+w_{s, s}^{(j)} \equiv 0, \quad s=0,1, \ldots, m-1 .
$$

Since $1 \leq j \leq N+1-n-m$, for all $N-n+1 \leq k \leq N$, we have

$$
j-k<j-k+1<\cdots<j-k+m-1<0,
$$

then, from (3.15) and (3.20), we deduce

$$
\tilde{y}_{k}^{(j)}=y_{t, k}^{(j)} \equiv 0, \quad N-n+1 \leq k \leq N, 1 \leq j \leq N+1-n-m .
$$

Proposition 3.5. The coefficients of order 0 in (3.19) satisfy the equation

$$
\tilde{y}_{k+m+n}^{(0)}+a_{m+n-1} \tilde{y}_{k+m+n-1}^{(0)} \cdots+a_{m} \tilde{y}_{k+m}^{(0)}=f_{k}, \quad k=0,1, \ldots, N-n-m .
$$

The coefficients of order $j, 1 \leq j \leq N-n-m+1$, in (3.19) satisfy the equation

$$
\begin{aligned}
\tilde{y}_{k+m+n}^{(j)} & +a_{m+n-1} \tilde{y}_{k+m+n-1}^{(j)}+\cdots+a_{m} \tilde{y}_{k+m}^{(j)} \\
= & -a_{m-1} \tilde{y}_{k+m-1}^{(j-1)}-a_{m-2} \tilde{y}_{k+m-2}^{(j-2)}-\cdots \\
& -a_{0} \tilde{y}_{k}^{(j-m)}, \quad k=0,1, \ldots, N-n-m .
\end{aligned}
$$

Proof. From (3.20), we get $\tilde{y}_{k}^{(j)}=y_{t, k}^{(j)}+w_{0, k}^{(j-k)}+w_{1, k}^{(j-k+1)}+\cdots+w_{m-1, k}^{(j-k+m-1)}$, that is,

$$
\tilde{y}_{k+m+l}^{(0)}=y_{t, k+m+l}^{(0)}, \quad \forall l \geq 0, k \geq 0 .
$$

Then (3.9) is equivalent to the difference equation (3.27). We show now that the coefficients $\tilde{y}_{k}^{(j)}, k=0, \ldots, N$, satisfy the difference equation given in (3.28).

First, notice that with the convention (3.13), we get a unique writing for (3.11) and (3.16), thus we does not have to consider several cases. If we replace the variable $j$ by $j+s-k-m$ in (3.16) that we multiply by -1 , we obtain

$$
\begin{aligned}
& w_{s, k+n+m}^{(j+s-k-n-m)}+a_{n+m-1} w_{s, k+n+m-1}^{(j+s-k-n-m+1)}+\cdots+a_{m} w_{s, k+m}^{(j+s-k-m)} \\
& =-a_{m-1} w_{s, k+m-1}^{(j+s-k-m)}-\cdots-a_{0} w_{s, k}^{(j+s-k-m)}, \quad k=0,1, \ldots
\end{aligned}
$$


Let us write (3.30) for various values of $s, s=0,1, \ldots, m-1$, the sum of these $m$ equations and (3.14) gives the following difference equation:

$$
\begin{aligned}
a_{m+n}\left(y_{t, k+m+n}^{(j)}+w_{0, k+m+n}^{(j-k-m-n)}+\cdots+w_{m-1, k+m+n}^{(j-k-n-1)}\right) & \\
& +a_{m+n-1}\left(y_{t, k+m+n-1}^{(j)}+w_{0, k+m+n-1}^{(j-k-m-n+1)}+\cdots+w_{m-1, k+m+n-1}^{(j-k-n)}\right) \\
& +\cdots+a_{m}\left(y_{t, k+m}^{(j)}+w_{0, k+m}^{(j-k-m)}+\cdots+w_{m-1, k+m}^{(j-k-1)}\right) \\
= & -a_{m-1}\left(y_{t, k+m-1}^{(j-1)}+w_{0, k+m-1}^{(j-k-m)}+\cdots+w_{m-1, k+m-1}^{(j-k-1)}\right) \\
& -\cdots-a_{0}\left(y_{t, k}^{(j-m)}+w_{0, k}^{(j-k-m)}+\cdots+w_{m-1, k}^{(j-k-1)}\right), \quad k=0,1,2, \ldots
\end{aligned}
$$

From (3.20), we make sure that (3.31) is nothing other than the difference equation (3.28).

Conclusion 3.6. Propositions 3.4 and 3.5 show that when $j \leq N+1-n-m$, the coefficients of expansion (3.8) satisfy the same problems given by our method in Section 3.1. By the uniqueness of the solutions of problems (3.3) and (3.4), we conclude that we have the same expansions of order $N+1-n-m$.

\section{Conclusion}

In this paper, we extended the perturbation method developed in [15], for a linear timevariant classical digital control system with several widely different coefficients. We considered a class of boundary value problems and we achieved two objectives. First, we gave sufficient conditions to ensure the existence and uniqueness of the solution of the boundary problem. Second, we gave an iterative method to get successive asymptotic approximations. This method is simple, straightforward, and convergent. It improves the singular perturbation method, indeed it offers considerable reduction and simplicity in computation since it does not require to compute boundary layer correction solutions. This method can be easily extended for initial value problems.

\section{References}

[1] C. Comstock and G. C. Hsiao, Singular perturbations for difference equations, The Rocky Mountain Journal of Mathematics 6 (1976), no. 4, 561-567.

[2] P. W. Eloe, Maximum principles for a family of nonlocal boundary value problems, Advances in Difference Equations 2004 (2004), no. 3, 201-210.

[3] J. Henderson, A. Peterson, and C. C. Tisdell, On the existence and uniqueness of solutions to boundary value problems on time scales, Advances in Difference Equations 2004 (2004), no. 2, 93-109.

[4] B. S. Kim, Y. J. Kim, and M. T. Lim, LQG control for nonstandard singularly perturbed discrete time systems, Proceedings of the 41st IEEE Conference on Decision and Control, Nevada, December 2002, pp. 3736-3741.

[5] _ Robust stabilizing control methods for singularly perturbed discrete bilinear systems, Proceedings of the 4th Asian Control Conference, Singapore, September 2002, pp. 670-675. 


\section{Perturbation method for difference equations}

[6] M. S. Krishnarayalu, Singular perturbation methods for one-point two-point and multi-point boundary value problems in multi-parameter digital control systems, Journal of Electrical and Electronics Engineering, Australia 19 (1999), 97-110.

[7] __ Singular perturbation methods for a class of initial and boundary value problems in multiparameter classical digital control systems, The ANZIAM Journal 46 (2004), no. 1, 67-77.

[8] B. A. Lawrence and B. Karna, An existence result for a multi-point boundary value problem on a time scale, to appear in Advances in Difference Equations.

[9] D. S. Naidu, Singular perturbations and time scales in control theory and applications: an overview, Dynamics of Continuous, Discrete \& Impulsive Systems. Series B. Applications \& Algorithms 9 (2002), no. 2, 233-278.

[10] D. S. Naidu and A. J. Calise, Singular perturbations and time scales in guidance and control of aerospace, Journal of Guidance, Control, and Dynamics 24 (2001), no. 6, 1057-1078.

[11] D. S. Naidu and A. K. Rao, Singular perturbation analysis of the closed-loop discrete optimal control problem, Optimal Control Applications \& Methods 5 (1984), no. 1, 19-37.

[12] Singular Perturbation Analysis of Discrete Control Systems, Lecture Notes in Mathematics, vol. 1154, Springer, Berlin, 1985.

[13] D. C. Popescu and Z. Gajić, Singular perturbation analysis of cheap control problem for sampled data systems, IEEE Transactions on Automatic Control 44 (1999), no. 11, 2209-2214.

[14] A. K. Rao and D. S. Naidu, Singular perturbation method applied to the open-loop discrete optimal control problem, Optimal Control Applications \& Methods 3 (1982), no. 2, 121-131.

[15] T. Sari and T. Zerizer, Perturbations for linear difference equations, Journal of Mathematical Analysis and Applications 305 (2005), no. 1, 43-52.

Tahia Zerizer: Laboratoire de Mathématiques, Département de Mathématiques, Université de Haute Alsace, 4 rue des Frères Lumière, Mulhouse 68093, France

E-mail address: t.zerizer@uha.fr 